\title{
Neutron-induced fission cross section on actinides using microscopic fission energy surfaces
}

\author{
M. Sin ${ }^{1, a}$, R. Capote ${ }^{2}$, S. Goriely ${ }^{3}$, S. Hilaire ${ }^{4}$, and A.J. Koning ${ }^{5}$ \\ 1 Nuclear Physics Department, University of Bucharest, Bucharest-Magurele, Romania \\ 2 Nuclear Data Section, International Atomic Energy Agency, 1400 Vienna, Austria \\ 3 Institut d'Astronomie et d'Astrophysique, Université Libre de Bruxelles, Campus de la Plaine, 1050 Brussels, Belgium \\ ${ }^{4}$ DPTA/Service de Physique Nucléaire, CEA/DAM Île-de-France, 91680 Bruyères-le-Châtel, France \\ 5 Nuclear Research and Consultancy Group, 1755 ZG Petten, The Netherlands
}

\begin{abstract}
Microscopic Hartree-Fock-Bogolyubov (HFB) calculations are now available and can provide all the nuclear ingredients required to describe the fission path from the equilibrium deformation up to the nuclear scission point. The aim of this paper is to apply the basic features of the optical model for fission, using the full microscopic information obtained from HFB models to calculate neutron-induced fission cross sections on selected actinide nuclei. This approach includes not only the details of the energy surface along the fission path, but also the estimate of the nuclear level density derived within the combinatorial approach on the basis of the same HFB single-particle properties, in particular at the fission saddle points. The sensitivity of the calculated fission transmission coefficients to different model approximations is studied and the predictive power of such a microscopic approach tested.
\end{abstract}

\section{Introduction}

Almost all existing evaluations of the neutron-induced fission cross section for nuclear energy applications rely on a multiple-humped fission barrier described by independent inverted parabolas. An advanced fission formalism, which uses the fission path parameterized as function of the quadrupole deformation by smoothly-joined parabolas, is the optical model for fission. By considering the existence of deformed class II states (and exotic hyper-deformed class III states in the case of light actinides) and their coupling with the normal states, an improved description of the fission probability can be achieved. Recently, the optical model for fission has been successfully applied to describe neutron-induced fission on Th-232 and Pa-231 nuclei [1]. However, fission barrier heights and widths are taken as free parameters, so that the predictive power of such approaches is relatively poor and they can not be used in applications requiring a blind description of fission for nuclei far from stability, such as nuclear astrophysics applications. An additional uncertainty arises from the wellestablished fact that microscopic estimates of the energy surfaces show a much more complex topology than the one possibly described by joined parabolas.

Comprehensive microscopic Skyrme Hartree-Fock-Bogolyubov (HFB) calculations [2] have now become available and can provide all the nuclear ingredients required to describe the fission path from the equilibrium deformation up to the nuclear scission. On the basis of the HFB nuclear ingredients, it is also possible to estimate the energy-, spin- and parity-dependent nuclear level density (NLD) derived within the combinatorial approach $[3,4]$, in particular at the fission saddle points.

The aim of this paper is to apply the basic features of the optical model for fission combined with the microscopic information obtained from HFB calculations to estimate the

\footnotetext{
${ }^{a}$ Presenting author, e-mail: mihaela.sin@gmail.com
}

neutron-induced fission cross sections on actinide nuclei. Calculations have been performed for almost all the isotopes of $\mathrm{Th}, \mathrm{Pa}, \mathrm{U}, \mathrm{Pu}, \mathrm{Np}, \mathrm{Am}, \mathrm{Cm}$ and $\mathrm{Cf}$ for which experimental data is available. The goal here is to assess the predictive power of HFB fission parameters and try to understand the reason for discrepancies with experimental data, and thus to generate a feedback needed for further improvements of the microscopic models and finally to produce recommendations on the fission parameters to be included in the next release of the RIPL database [5]. In the following sections, a representative selection of calculated cases together with the fission formalism used in the calculations and a critical analysis of the results is given.

\section{Global microscopic fission parameters}

The full 3-dimensional (3-D) energy surfaces have been calculated for $80 \leq Z \leq 98$ nuclei on the basis of the microscopic Skyrme Hartree-Fock-Bogolyubov approach [2]. The Skyrme force used to estimate the fission properties corresponds to BSk14 which was fitted to all known nuclear masses and nuclear matter properties. In addition, the corresponding HFB14 mass model has been determined by including a phenomenological vibrational correction to be able to reproduce at best the energy surface at large deformation, hence the fission properties. In particular, the rms deviation for the 52 known primary barriers [5] of nuclei with $88 \leq \mathrm{Z} \leq 96$, which are always less than $9 \mathrm{MeV}$ high, is $0.67 \mathrm{MeV}$. A similar accuracy is obtained $(0.65 \mathrm{MeV})$ for the secondary barriers. The fission path considered in the present calculation is obtained by projecting the 3-D energy surface along the quadrupole deformation parameter. The fission path corresponds to the most gently climbing or steepest descending static path found. Illustrations are given in [2].

Energy-, spin- and parity-dependent NLD have been also calculated using the combinatorial model of ref. $[3,4]$. In 


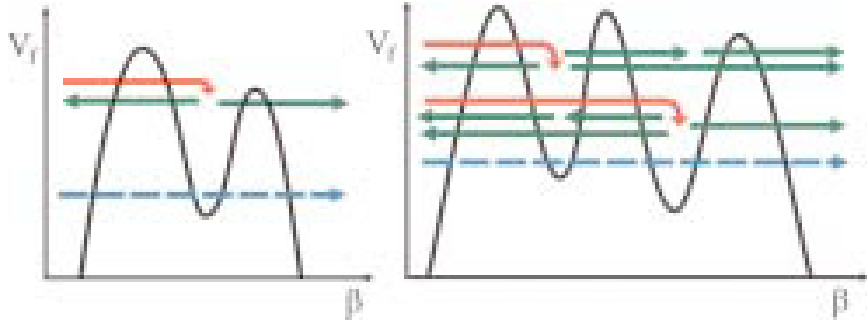

Fig. 1. Illustration of the transmission mechanisms through fission barriers: direct transmission (dashed arrows) and absorption into well(s) followed by emission in the fission channel or shape transition(s) to class I (II) states (full arrows).

such an approach, the microscopic intrinsic state density and rotational enhancement factor are estimated consistently on the basis of the microscopic HFB single-particle and pairing properties, but the vibrational modes remain treated on a phenomenological footing. It is also important to note that calculated fission NLD do not include enhancements due to axial/reflection asymmetry of the nuclear shape at saddle points. The reflection asymmetry at the outer saddle was taken into account by multiplying the tabulated NLD by the enhancement factor $K_{B}=2$. For quantitative assessments, an average enhancement factor $K_{A}=10$ was applied for axial asymmetric shapes at the inner saddle point.

\section{Fission model}

The fission model used in present calculations has two unique features:

- partial damping of the vibrational states in the fission barrier wells;

- numerical fission barriers along the fission path.

Depending on the degree of damping of the vibrational states in the potential minima, fission occurs either by direct transmission through the barrier or by reemission into the fission channel after absorption into the isomeric well (as long as the delayed fission is neglected). The fission transmission coefficients associated with these two mechanisms (schematically illustrated in fig. 1) are the direct transmission coefficient $\left(T_{\text {dir }}\right)$ and the indirect $\left(T_{\text {ind }}\right)$ one, respectively, their sum representing the total fission coefficient $\left(T_{f}\right)$.

At very low excitation energies, where the vibrational states in the potential wells preserve their individuality, fission proceeds entirely by direct transmission across the barrier. In the other extreme case, i.e., for a complete damping which holds for excitation energies close or above the top of the barrier, the entire flux transmitted through the inner hump(s) is absorbed into the well(s) and the fission is completely determined by the indirect mechanism.

In the intermediate case, the fission process can be described by a smooth transition between the direct and indirect transmissions. In the optical model for fission this is achieved by introducing a negative imaginary potential for the deformation range corresponding to the absorbing wells. A simpler way to describe the degree of damping is to introduce an energy-dependent quantity $w$, which ranges from zero to one [6]. The $w=0$ value corresponds to the zero damping at the excitation energy equal to the bottom of the well; $w=1$ corresponds to the full damping at the excitation energy equal to the top of the barrier. The total fission coefficient is obtained as a weighted sum of the direct $\left(T_{\text {dir }}\right)$ and indirect $\left(T_{\text {ind }}\right)$ contributions reading

$$
T_{f}=\left(1-w_{1}\right) T_{d i r}+w_{1} T_{\text {ind }_{1}}
$$

for the double-humped fission barrier and

$$
T_{f}=\left(1-w_{1}\right) T_{d i r}+w_{1} T_{i n d_{1}}+\left(1-w_{1}\right) w_{2} T_{i n d_{2}}
$$

for a triple-humped fission barrier. The direct transmission coefficient for a zero damping case is calculated as in ref. [1]. The indirect transmission coefficient in the full damping limit is described by the well-known expression

$$
\frac{1}{T_{f}}=\sum_{j} \frac{1}{T_{j}}
$$

where $T_{j}$ are the transmission coefficients through humps and $j$ runs from 1 to the number of humps. The single-hump transmission coefficient reads

$$
T_{j}=\frac{1}{1+\exp \left(2 M_{j}\right)}
$$

being $M_{j}$ the momentum integral for the hump, expressed in the first-order WKB approximation as [1]:

$$
M_{j}= \pm\left|\int_{a_{j}}^{b_{j}}\left[2 \mu\left(E-V_{j}(\beta)\right) / \hbar^{2}\right]^{1 / 2} d \beta\right|
$$

where the + sign is taken when the excitation energy is lower than the hump under consideration and the - when it is higher. In the above equation, $E$ is the excitation energy, $a_{j}$ and $b_{j}$ are the deformations corresponding to the intercepting points, $\mu$ is the inertial mass parameter, assumed here to be independent of the deformation $\beta$ and approximated by the semi-empirical expression $\mu \approx 0.054 A^{5 / 3} \mathrm{MeV}^{-1}$, where $A$ is the mass number. In our calculations, $V(\beta)$ is the tabulated HFB potential energy along the fission path as a function of the quadrupole deformation $\beta$. In the case of a single parabolic barrier, combining formulae (4) and (5) yields the well-known Hill-Wheeler transmission coefficient.

In the present calculations the transition states spectra are continuous and described by NLD. We could easily consider transitional discrete states [1], but there is at the present time no global prediction for collective low-lying discrete transition states available. The resonant structure appearing near the fission threshold [1] can therefore not be described. Finally, the total fission transmission coefficient through hump $j$ for an energy $E$, a spin $J$ and parity $\pi$ is given by

$$
T_{j}(E, J, \pi)=\int_{0}^{\infty} \frac{\rho_{j}(\varepsilon, J, \pi)}{1+\exp \left(2 M_{j}\right)} d \varepsilon .
$$

\section{Results and discussions}

The calculations have been performed with the updated version of the nuclear reaction code EMPIRE 2.19 [7,8]. The 


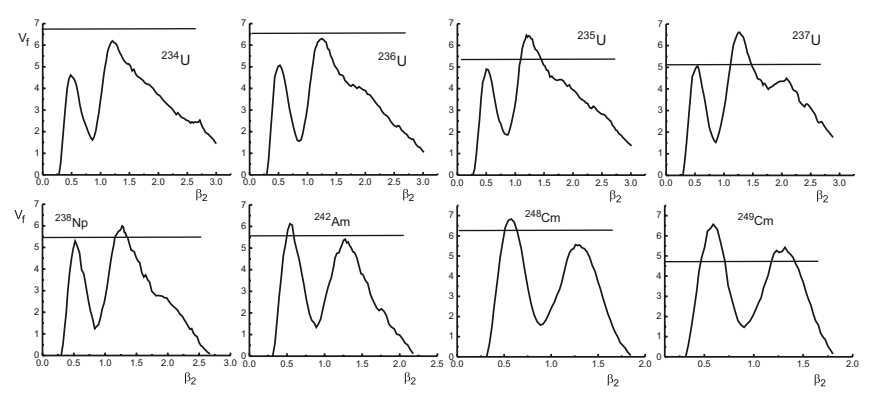

Fig. 2. HFB fission barriers for the studied nuclei [3]. The horizontal line indicates the neutron separation energy.

regional parameterization of the neutron dispersive coupledchannel optical model potential (RIPL 2408) [9] has been used for actinide nuclei. Hauser-Feshbach and Hofmann-RichertTepel-Weidenmuller versions of the statistical model were used for the compound nucleus cross section calculations, including the full gamma-cascade.

The following input parameters have been adopted:

- discrete level schemes (RIPL-2 [5]);

- HFB NLD for normal states [3,4];

- gamma strength functions MLO (RIPL-2 [5]);

- HFB numerical fission barriers [2];

- HFB NLD at saddles [3,4].

Some static one-dimensional fission barriers used in the present calculations are shown in figure 2. For such nuclei, above the neutron separation energy, both humps are well approximated by inverted parabolas. Our tests indicate that for neutron-induced fission cross section calculations, parabolic barriers could be used both to keep the coding simple, and to speed up calculations. However, for photon and chargedparticle induced fission, significant deviations appear between the transmission through the real and the parabolic barriers. The shape of the barrier (especially its width) is also crucial for the correct prediction of the spontaneous fission half life and the half-life of shape-isomers. Important deviations with respect to the parabolic shape can also be expected for neutron-rich nuclei [3].

Figures 3-6 compare the calculated and experimental neutron-induced fission cross section (left panel) for U-233, U-235, U-234 and U-236. The NLD at saddle points (A and $\mathrm{B}$ (and $\mathrm{C}$ for U-236)) are shown in the right panel. The fission cross sections exhibit two similar features, namely a slight or severe underestimate of the experimental data, and some discontinuities in the energy range up to $2 \mathrm{MeV}$ (corresponding to the excitation energy up to $2 \mathrm{MeV}$ above the fission barrier). As shown by the dashed curves, such an underestimation of the experimental data is essentially due to the missing enhancement factor $K_{B}$ affecting NLD for asymmetric shapes, and the overestimate by a few hundred $\mathrm{keV}$ of the fission barrier height by the HFB model. The discrepancies in the cross section prediction are particularly severe near the fission threshold in the case of fertile isotopes. The up (down) arrows in figures 3-4 indicate the corresponding excitation energy above the top of the inner (outer) hump. As expected, the fissile isotopes are more sensitive to NLD, while the fertile are more sensitive to fission barrier parameters. By changing the height of the highest ( $V_{B}$ in this case) hump by a quantity
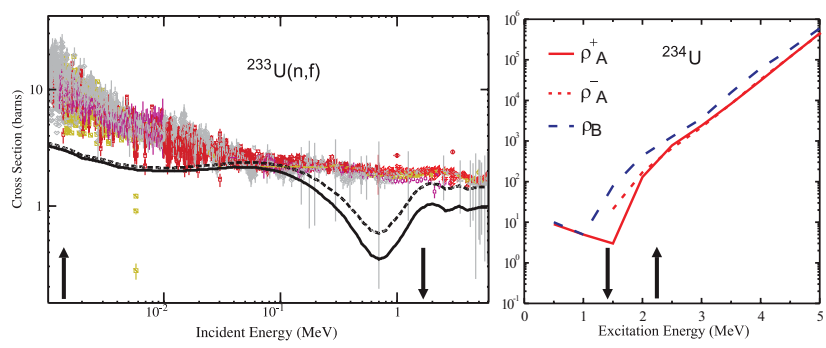

Fig. 3. ${ }^{233} U(n, f)$ cross sections (full curve: default parameters, dashed curve: outer saddle symmetry enhancement $K_{B}=2$ ).

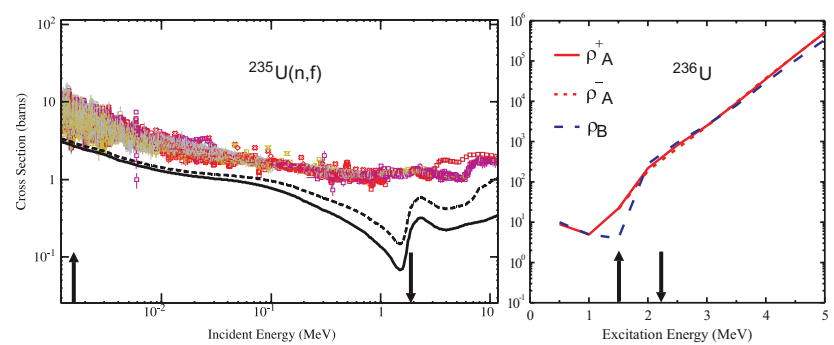

Fig. 4. ${ }^{235} \mathrm{U}(\mathrm{n}, \mathrm{f})$ cross sections (full curve: default parameters, dashed curve: $\left.K_{B}=2\right)$.

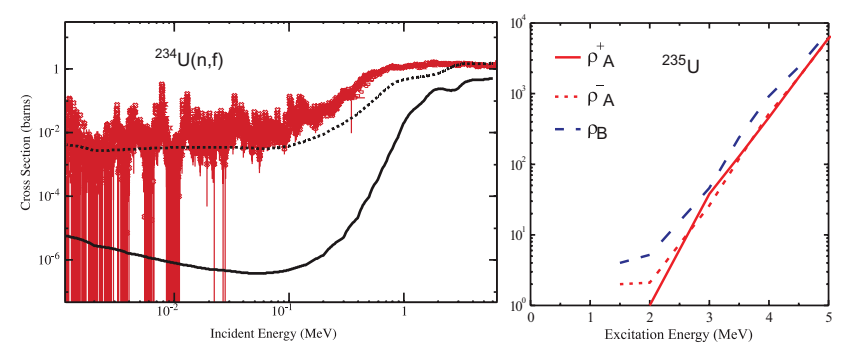

Fig. 5. ${ }^{234} \mathrm{U}(\mathrm{n}, \mathrm{f})$ cross sections (full curve: original parameters, dashed curve: $\left.K_{B}=2, V_{B}-0.7 \mathrm{MeV}\right)$.
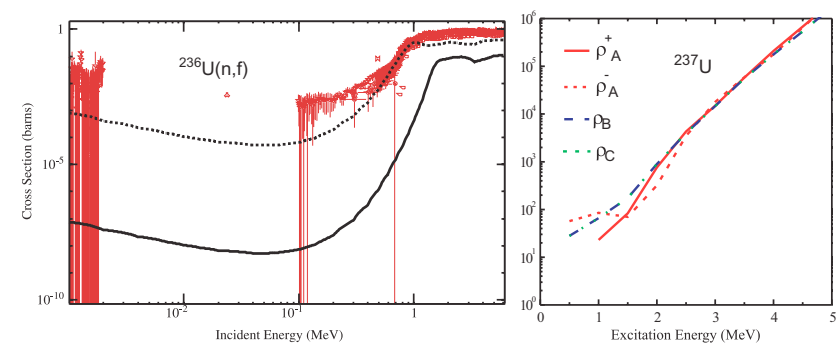

Fig. 6. ${ }^{236} \mathrm{U}(\mathrm{n}, \mathrm{f})$ cross sections (full curve: original parameters, dashed curve: $K_{B}=2, V_{B}-0.6 \mathrm{MeV}$ ).

within the HFB uncertainty (figs. 5-6) a better description of the threshold can be recovered for fertile isotopes.

The discontinuities mentioned above are directly related to the NLD behaviour below $2 \mathrm{MeV}$. It reflects the missing low-lying collective states, which are expected to be located within the pairing gap and are not described by the present model $[3,4]$. This is the reason why the observed problem is common to all even- $\mathrm{Z}$ isotopes ( $\mathrm{U}, \mathrm{Pu}$ and $\mathrm{Cm})$. In fact, the biggest gap in the fission NLD is observed in the odd isotopes of the even-Z nuclei like U-233, U-235 and Cm-247. Indeed the compound fissioning nucleus is even-even in those cases and the pairing effect is strong. On the other hand, 

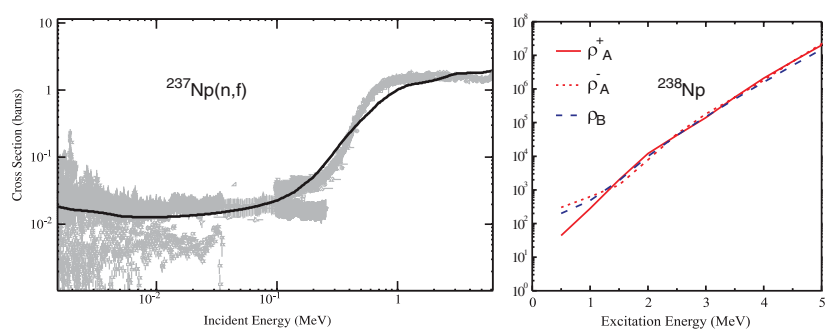

Fig. 7. ${ }^{237} \mathrm{~Np}(\mathrm{n}, \mathrm{f})$ cross sections with original parameters.

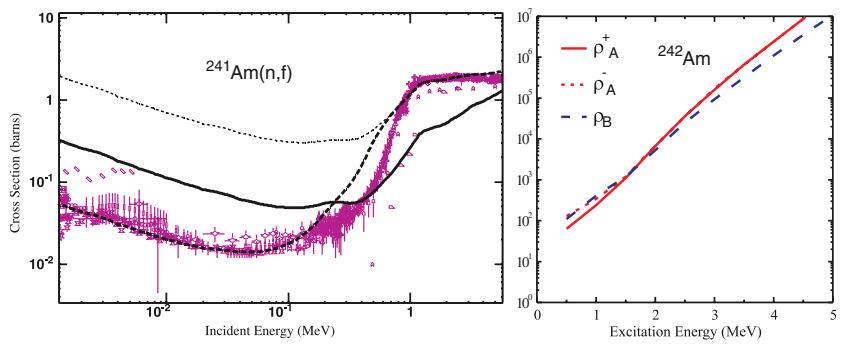

Fig. 8. ${ }^{241} \mathrm{Am}(\mathrm{n}, \mathrm{f})$ cross sections (full curve: original parameters, dotted curve: $K_{A}=10, K_{B}=2$, dashed curve: partial damping).
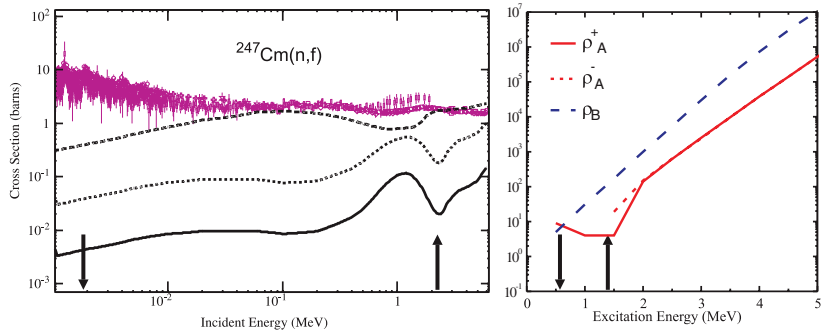

Fig. 9. ${ }^{247} \mathrm{Cm}(\mathrm{n}, \mathrm{f})$ cross sections (full curve: original parameters, dotted curve: $K_{A}=10, K_{B}=2$, dashed curve: $V_{A}-0.8 \mathrm{MeV}$ ).

we expect almost no pairing effect for odd- $Z$ odd-A nuclei like Np-237 and Am-241 since, in this case, the fissioning compound nucleus is odd-odd. As can be observed in figures $7-8$, the agreement with experimental data is indeed much better for Np-237 and Am-241.

The excellent description of the experimental data shown in figure 7 for the ${ }^{237} \mathrm{~Np}(\mathrm{n}, \mathrm{f})$ reaction deserves further comments as far as the symmetry of this nucleus at the inner saddle point is concerned. In the case of ${ }^{241} \mathrm{Am}$ (fig. 8) the height of the fission barrier is in agreement with the experimental threshold. The full curve obtained with the original parameters does not follow the trend of the experimental data, but is close to them. By introducing average values for the nuclear shape asymmetry enhancement factors $\left(K_{A}=10, K_{B}=2\right)$, a good description of the data above $1 \mathrm{MeV}$ and a strong overestimate below the threshold are obtained (dotted curve). This behaviour is a typical one when full damping is assumed at sub-barrier excitation energies. By applying the formulae for the partial damping introduced in section 3 , a good agreement with experimental data is obtained in the whole energy region (see dashed line in fig. 8).

Neutron-induced fission cross sections on $\mathrm{Cm}$ isotopes are shown in figures 9-10. Results are similar to those found for the $\mathrm{U}$ isotopes (figs. 3-6) as curium also has an even number of protons.

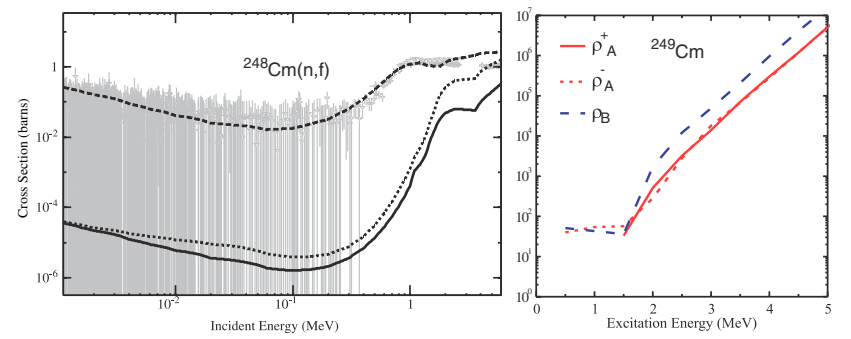

Fig. 10. ${ }^{248} \mathrm{Cm}(\mathrm{n}, \mathrm{f})$ cross sections (full curve: original parameters, dotted curve: $K_{A}=10, K_{B}=2$, dashed curve: $V_{A}-1.0 \mathrm{MeV}$ ).

\section{Conclusions}

Preliminary results for the calculations of neutron-induced fission cross section, using the new HFB global microscopic parameters are encouraging. As expected, the accuracy required in nuclear applications is not reached yet.

An extensive analysis revealed two main causes of discrepancies between calculations and measurements in the energy range of $0.001-5.0 \mathrm{MeV}$. The first is related to the barriers' height, which for most of the cases studied here are too high by some $0.5-1 \mathrm{MeV}$ with respect to experimental data (these differences are within the HFB uncertainty). The second cause is due to the NLD behaviour at low energies. The introduction of low-lying collective discrete states within the pairing gap by a convolution of collective and single-particle states [10] should improve the situation. More reliable conclusions are expected after asymmetry NLD enhancements for both the inner and outer barriers are considered.

A new fission formalism representing an alternative to the optical model for fission has been formulated. It allows for a smooth transition from the direct to the indirect transmission mechanism, is valid for any excitation energy both for discrete states and the continuum, and is capable of fully exploiting the information produced by microscopic HFB calculations. By combining a realistic fission model with global microscopic parameters, a significant step towards fission prediction has been made.

\section{References}

1. M. Sin et al., Phys. Rev. C 74, 014608 (2006).

2. S. Goriely, J.M. Pearson (these proceedings).

3. S. Hilaire, S. Goriely (these proceedings).

4. S. Hilaire, S. Goriely, Nucl. Phys. A 779, 63 (2006).

5. T. Belgya, O. Bersillon, R. Capote et al., Handbook for calculations of nuclear reaction data: Reference Input Parameter Library-2, Technical Report IAEA-TECDOC-1506 (IAEA, Vienna, Austria, 2006). Available online @ http://wwwnds.iaea.org/RIPL-2/.

6. M. Sin, R. Capote (to be published).

7. M. Herman, P. Obložinský, R. Capote, M. Sin, A. Trkov, A. Ventura, V. Zerkin, in Proceedings of the International Conference on Nuclear Data for Science and Technology, 27 Sept.-1 Oct. 2004, Santa Fe, NM USA.; AIP Conf. Proc. 769, 1184 (2005). Online @ www.nndc.bnl.gov/empire219/.

8. R. Capote, M. Sin, A. Trkov, M. Herman, B.V. Carlson, P. Obložinský (these proceedings).

9. R. Capote et al., Phys. Rev. C 72, 064610 (2005).

10. R. Capote et al., Phys. Rev. C 71, 064320 (2005). 